\title{
Adrenalectomía bilateral laparoscópica por metástasis asincrónica de melanoma: comunicación de un caso con sobrevida prolongada*
}

\author{
Drs. ROBERTO MOLINA E. ${ }^{1}$, ORLANDO ORDOÑEZ P. ${ }^{2}$, OCTAVIO A. CASTILLO C. ${ }^{3}$
}

\author{
Servicio de Urología. Hospital Universitario de Fuenlabrada. Madrid, España. \\ Caja Costarricense de Seguro Social, San José, Costa Rica. \\ 3 Departamento de Urología y Centro de Cirugía Robótica, Clínica INDISA y Facultad de Medicina, \\ Universidad Andrés Bello, Santiago, Chile.
}

\begin{abstract}
\section{Bilateral adrenalectomy for asynchronous metastases of a malignant melanoma}

Clinical case: We report a 70 years old male with a history of an ear lobe melanoma with was excised seven years ago, who had a bronchial relapse and required a right pneumonectomy. During a follow up abdominal CAT scan, a $9 \mathrm{~cm}$ tumor in the left adrenal gland was detected. The patient was operated, performing a left adrenalectomy and nephrectomy. The pathologic study confirmed the presence of a fusocellular melanoma. One year later, a right adrenal mass was detected and excised. The pathological study of the piece again confirmed a metastasis of a malignant melanoma. The patient died due to progression of the disease, 10 years after the adrenalectomy.
\end{abstract}

Key words: Adrenal metastases, laparoscopic adrenalectomy, melanoma.

\section{Resumen}

Objetivo: Presentar un caso de metástasis suprarrenal bilateral asincrónica de Melanoma cutáneo tratado con adrenalectomía laparoscópica bilateral. Caso clínico: Paciente de 70 años con antecedente de melanoma del pabellón auricular extirpado 7 años antes de su consulta urológica. Posteriormente, presenta una recidiva bronquial tratada con quimioterapia, radioterapia y neumonectomía derecha. En sus exámenes de seguimiento una Tomografía computada muestra el hallazgo incidental de una lesión tumoral de $9 \mathrm{~cm}$ en la glándula suprarrenal izquierda. Se realizó nefrectomía y adrenalectomía izquierda laparoscópica en bloque sin incidencias. El análisis histopatológico confirmó el hallazgo de una metástasis de melanoma fuso-celular. Un año después el paciente presenta un nuevo hallazgo incidental de un tumor de $3 \mathrm{~cm}$ en la glándula suprarrenal derecha, la cual fue tratada con adrenalectomía laparoscópica, y cuyo análisis histopatológico demostró metástasis de melanoma maligno. El paciente fallece por progresión de su enfermedad 10 años después de su cirugía suprarrenal. Conclusiones: En los pacientes con metástasis suprarrenal de melanoma, la adrenalectomía incrementa la supervivencia cáncer especifica en relación a los pacientes tratados sin cirugía. El abordaje laparoscópico constituye una alternativa terapéutica con menor morbilidad que la cirugía abierta en cirujanos con experiencia laparoscópica.

Palabras clave: Metástasis suprarrenal, adrenalectomía laparoscópica, melanoma.

*Recibido el 10 de diciembre de 2012 y aceptado para publicación el 14 de enero de 2013.

Los autores no refieren conflictos de interés

Correspondencia: Dr. Octavio A. Castillo C.

Av. Santa María 1810, Santiago, Chile. CP 7520440.

octavio.castillo@indisa.cl 


\section{Introducción}

El melanoma maligno es el quinto tumor más frecuente en los varones y el sexto en las mujeres en Estados Unidos. Conjuntamente con el cáncer de pulmón, riñón, mama y tiroides presenta una gran tendencia a producir metástasis en la glándula suprarrenal' ${ }^{1}$ Según la clasificación de la American Joint Committee on Cancer Union Against Cancer (AJCC), la presencia de metástasis, tanto en el momento del diagnóstico como en el seguimiento, se considera como estadio IV-M1, correspondiendo el M1a a las lesiones en piel, tejidos blandos y ganglios linfáticos, M1b a las metástasis pulmonares y M1c a metástasis viscerales distintas de las anteriores ${ }^{2}$.

El compromiso metastásico de la glándula suprarrenal en esta neoplasia se encuentra entre el 36 y $54 \%$ en las series de autopsia, aunque su presencia estimada en el seguimiento clínico de los pacientes con melanoma es solamente del $1 \%$, dificultada fundamentalmente por la ausencia de síntomas ${ }^{3}$.

El tratamiento del melanoma en estadio IV es controvertido. Las terapias sistémicas como las interleukinas y la decarbazina, tanto solas como en combinación, consiguen tasas de supervivencia a cinco años inferiores al 5\%, siendo el principal factor pronóstico de supervivencia la respuesta clínica completa ${ }^{2}$.

Dada la resistencia del tumor a los esquemas farmacológicos, el tratamiento del melanoma metastásico ha experimentado un cambio en los últimos años, siendo el abordaje quirúrgico con resección con márgenes libres del tumor una alternativa terapéutica adecuada para casos seleccionados. Con esta conducta se ha logrado un aumento de la sobrevida hasta un $42 \%$ a cinco años en tumores $\mathrm{M}^{3} \mathrm{a}^{3}$.

Si bien el manejo laparoscópico de las lesiones suprarrenales malignas, puede ser tema de controversia, nuestra experiencia demuestra que en centros de alto volumen, es una alternativa segura ${ }^{4}$.

Presentamos un caso de extirpación laparoscópica adrenal bilateral de metástasis metacrónicas en la glándula suprarrenal de melanoma maligno cutáneo. Después de la revisión bibliográfica efectuada en los buscadores médicos PUBMED y Medline, creemos que es el primer caso descrito en la literatura hispano-americana hasta la fecha.

\section{Caso clínico}

Paciente de 70 años con el antecedente de melanoma del pabellón auricular derecho extirpado 7 años antes de la consulta urológica. Seis años después presenta recidiva bronquial derecha tratada satisfactoriamente mediante inmunoterapia, quimioterapia, radioterapia y neumonectomía derecha.
Un año después se diagnostica una metástasis en zona de carina traqueal y muñón bronquial derecho tratadas con electrofulguracion a través de broncoscopia rígida y otra en la glándula suprarenal izquierda de $9 \mathrm{~cm}$ de diámetro máximo.

En relación a la lesión adrenal se propone la realizacion de exéresis por vía laparoscópica. La intervención se realiza mediante acceso transperitoneal, siendo necesaria la extirpación adrenal y del riñón izquierdo en bloque por encontrarse la glándula englobada por los vasos renales (Figura 1), llevándose a cabo sin incidencias intraoperatorias. La anatomía patológica demostró una masa suprarrenal izquierda que engloba el hilio renal y que en conjunto pesan $568 \mathrm{~g}$. La masa adrenal pesa $156 \mathrm{~g}$ y mide 9 x $5 \mathrm{~cm}$ y el estudio histológico confirma una metástasis masiva de neoplasia maligna pobremente diferenciada con los caracteres de melanoma principalmente fusocelular y parcialmente necrosado, con márgenes negativos y ganglios linfaticos regionales negativos para metástasis.

Seis meses después en el control posterior por TC aparece una lesion nodular de $3 \mathrm{~cm}$ en la glándula suprarrenal derecha, por lo que se realiza adrenalectomía laparoscópica transperitoneal derecha, sin incidentes, confirmando el estudio histológico la presencia de una metástasis nodular de melanoma maligno de $3 \times 2 \mathrm{~cm}$, principalmente localizado en la médula adrenal, con márgenes quirúrgicos negativos (Figura 2).

El paciente fallece por progresión de la enfermedad 10 años después de la primera cirugía suprarrenal, a la edad de 80 años.

\section{Discusión}

El pronóstico oncológico del melanoma en estadio IV es desfavorable, aunque es distinto para cada uno de los subgrupos de la AJCC, siendo más favorable en los casos sin evidencia de afectación visceral (M1a). En los casos con evidencia de afectación de órganos sólidos, el pronóstico es mejor para las metástasis pulmonares (M1b) que para el resto de órganos (M1c). Sin embargo, la resección completa de las lesiones, entendida como la exéresis de las metástasis clínicas y radiológicas con márgenes libres, puede mejorar la supervivencia de estos pacientes ${ }^{5}$.

La presencia de metástasis de melanoma en la glándula suprarenal, es una entidad diagnosticada durante el seguimiento de estos tumores de forma incidental puesto que la presencia de síntomas como dolor o sangrado retroperitoneal es poco frecuente. En la TC, estas masas, son con frecuencia heterogéneas, voluminosas y engloban órganos vecinos ${ }^{3}$.

En el trabajo de Haigh y cols ${ }^{6}$, de 83 pacientes 


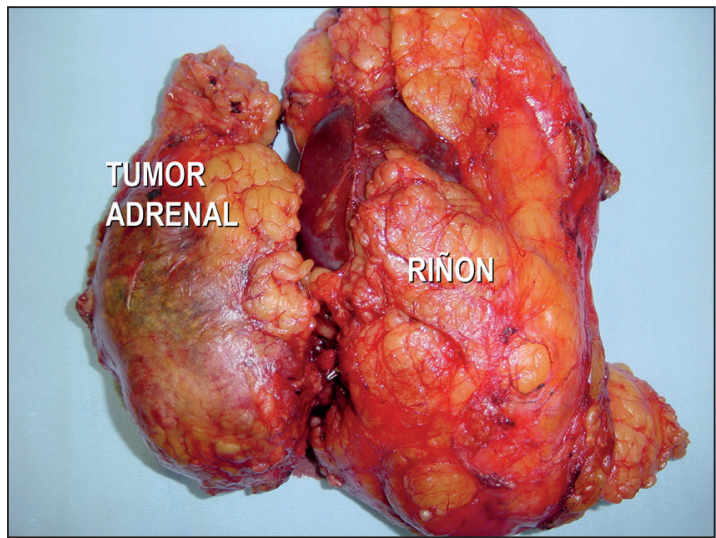

Figura 1. Adrenalectomía + nefrectomía en bloque izquierdo.

con metástasis adrenales por melanoma, 27 fueron intervenidos quirúrgicamente, 18 con intención curativa, todos mediante cirugía abierta. La supervivencia media de todos los pacientes fue de 9,3 meses, siendo de 25,7 meses en los operados con intención curativa.

En la serie de Collinson y cols ${ }^{5}$, con 186 pacientes con afectación metastásica de la glándula suprarenal por melanoma cutáneo, sólo 23 fueron intervenidos quirúrgicamente por vía abierta, con intención curativa presentando una supervivencia media de 16 meses (rango de 1-55 meses) frente a 5 meses en los pacientes no intervenidos. La recurrencia adrenal fue la localización inicial en 9 de los pacientes intervenidos, en los 14 pacientes restantes, al igual que en nuestro caso, existió una recidiva previa intervenida quirúrgicamente.

Branum y cols ${ }^{7}$, en su serie de 10 melanomas con afectación suprarenal, 8 fueron intervenidos quirúrgicamente consiguiendo una supervivencia media de 59 meses.

Estos trabajos analizan los factores predictores de supervivencia cáncer específica en pacientes con metástasis adrenales de melanoma cutáneo. La resección completa de las metástasis adrenales y un período libre de enfermedad prolongado entre el tratamiento del melanoma y la aparición de metástasis suprarrenal son los principales factores responsables de una mayor supervivencia cáncer específica. En el trabajo de Collinson ${ }^{5}$, los pacientes con resescción completa y períodos libres de enfermedad superiores al año, presentaron una supervivencia mayor que los que desarrollaron la metástasis adrenal en menos de un año del diagnóstico inicial del melanoma. Este hecho se da también en los pacientes con metástasis suprarenales de otros tumores distintos del melanoma como demuestran Sarela y $\operatorname{cols}^{8}$, en su trabajo de

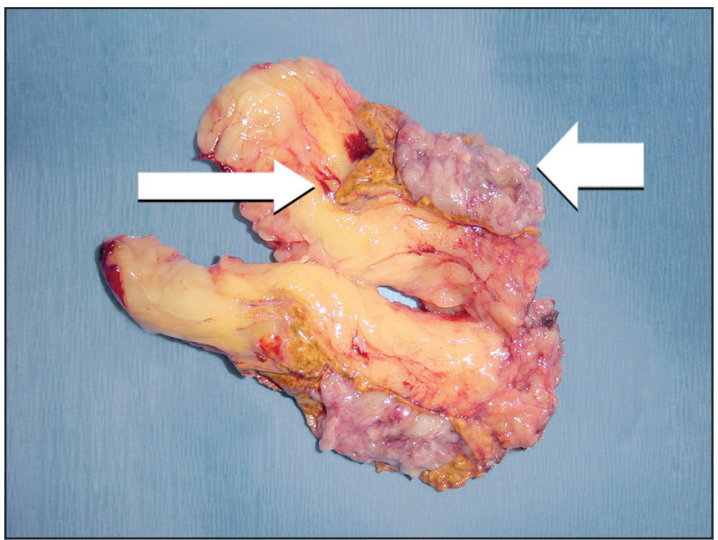

Figura 2. La flecha gruesa muestra la metástasis y la flecha angosta parte de la glándula suprarrenal normal.

41 pacientes sometidos a adrenalectomía, encontrando una mayor supervivencia en los que presentaban períodos libres de enfermedad superiores a seis meses independientemente de la estirpe tumoral.

La explicación más probable es que la presencia de intervalos libres de enfermedad largos se asocia a una menor agresividad biológica del tumor, con un tiempo prolongado de baja actividad hasta la recidiva y cuando aparece se comportará del mismo modo que la lesión primaria; y que los pacientes sometidos a adrenalectomía por metástasis corresponden generalmente a casos muy seleccionados con buen estado general y poca carga tumoral asociada a la lesión suprarrenal.

El desarrollo de las técnicas laparoscópicas ha representado un cambio importante en todos los aspectos relacionados a la cirugía de la glándula suprarrenal. Tres tipos de abordaje laparoscópico han sido descritos: anterior, lateral y posterior. La adrenalectomía laparoscópica se considera el estándar de oro para el tratamiento de las lesiones benignas así como de las lesiones metastásicas de la glándula, pero su valor en tumores malignos primarios y las lesiones metastásicas adrenales está todavía en discusión debido al gran tamaño que pueden llegar a alcanzar y el compromiso de órganos vecinos que implique la necesidad de grandes resecciones ${ }^{9}$. En el trabajo publicado por Castillo y cols ${ }^{4}$, se realizaron 34 adrenalectomías laparoscópicas por metástasis de diversos tumores. Todas se realizaron por vía transperitoneal lateral sin necesidad de conversión a cirugía abierta ni complicaciones intraoperatorias en ningún caso, con un tiempo quirúrgico medio de 87 min y una pérdida sanguínea media inferior a $100 \mathrm{cc}$. Este trabajo revela, por lo tanto, una ventaja en cuanto al sangrado, complicaciones, estancia hospitalaria y período de convalecencia en relación 
a la cirugía convencional abierta, siendo necesaria una gran experiencia en cirugía laparoscópica para su realización.

En nuestro caso la cirugía pudo realizarse por vía laparoscópica, obteniéndose una supervivencia a largo plazo mayor de la observada en las series previas.

\section{Conclusiones}

La afectación de la glándula suprarrenal en el melanoma estadio IV es infrecuente y su pronóstico en los pacientes tratados exclusivamente con terapia sistémica, es muy adverso con una supervivencia inferior al $10 \%$ a cinco años.

La presencia de intervalos libres de enfermedad prolongados, desde el diagnóstico primario hasta el desarrollo de la metástasis y la resección completa de la misma, constituyen los dos factores más importantes en la supervivencia cáncer específica.

La vía laparoscópica constituye una alternativa terapéutica a la cirugía convencional abierta en el tratamiento de las metástasis adrenales, aportando una menor morbilidad que la cirugía convencional abierta, cuando se realiza por cirujanos con experiencia laparoscópica.

\section{Referencias}

1. Bertagna F, Biasiotto G, Rodella C, Werner T, Giubbini R, Alavi A. Massive bilateral adrenal gland metastases from Melanoma diagnosed by F18-FDG-PET/CT. Jpn J Radiol. 2009;27:392-3.
2. Ollila D. Complete metastasectomy in patients with stage IV metastatic Melanoma. Lancet Oncol. 2006; 7:91924

3. Cuesta Alcala JA, Caballero Martínez MC, Ripa Saldías L, Pascual Piedrola I, Solchaga Martínez A, Aldave Villanueva $\mathrm{J}$, et al. Therapeutic approach in adrenal Melanoma. Review of the literature. Arch Esp Urol. 2001;54:685-90.

4. Castillo OA, Vitagliano G, Kerkebe M, Parma P, Pinto I, Díaz M. Laparoscopic adrenalectomy for suspected metastasis of adrenal glands: our experience. Urology 2007;69:637-41.

5. Collinson FJ, Lam TK, Bruijn WM, de Wilt JH, Lamont M, Thompson JF, Kefford RF. Long-term survival and occasional regression of distant Melanoma metastases after adrenal metastasectomy. Ann Surg Oncol. 2008;15:1741-9.

6. Haigh PI, Essner R, Wardlaw JC, Stern SL, Morton DL. Long-term survival after complete resection of Melanoma metastatic to the adrenal gland. Ann Surg Oncol. 1999;6:633-9.

7. Branum GD, Epstein RE, Leight GS. The role of resection in the management of Melanoma metastatic to the adrenal Gland. Surgery 1991;109:127-31.

8. Sarela AI, Murphy I, Coit DG, Conlon K. Metastasis to the adrenal gland: the emerging role laparoscopic surgery. Ann Surg Oncol. 2003;10:1191-6.

9. Carlini M, Lonardo MT, Boschetto A, Carboni F, Appetecchia M, Tropea F, et al. Adrenal glands metastases from malignant Melanoma. Laparoscopic bilateral adrenalectomy. J Exp Clin Cancer Res. 2003; 22:141-5. 